\title{
Hierarchical Sliding Mode Algorithm for Athlete Robot Walking
}

\author{
Van Dong Hai Nguyen, ${ }^{1}$ Xuan-Dung Huynh, ${ }^{1}$ Minh-Tam Nguyen, ${ }^{1}$ \\ Ionel Cristian Vladu, ${ }^{2}$ and Mircea Ivanescu ${ }^{2}$ \\ ${ }^{1}$ Ho Chi Minh City University of Technology and Education (HCMUTE), 01 Vo Van Ngan Street, Thu Duc District, \\ Ho Chi Minh City, Vietnam \\ ${ }^{2}$ University of Craiova (UCV), 13 Alexandru Ioan Cuza Street, 200585 Craiova, Romania
}

Correspondence should be addressed to Van Dong Hai Nguyen; hainvd@hcmute.edu.vn

Received 4 July 2017; Revised 26 October 2017; Accepted 12 November 2017; Published 6 December 2017

Academic Editor: Yangmin Li

Copyright (C) 2017 Van Dong Hai Nguyen et al. This is an open access article distributed under the Creative Commons Attribution License, which permits unrestricted use, distribution, and reproduction in any medium, provided the original work is properly cited.

Dynamic equations and the control law for a class of robots with elastic underactuated MIMO system of legs, athlete Robot, are discussed in this paper. The dynamic equations are determined by Euler-Lagrange method. A new method based on hierarchical sliding mode for controlling postures is also introduced. Genetic algorithm is applied to design the oscillator for robot motion. Then, a hierarchical sliding mode controller is implemented to control basic posture of athlete robot stepping. Successful simulation results show the motion of athlete robot.

\section{Introduction}

Two-legged robot is an interesting topic which lasts for long time [1-3]. One of the classical forms of this type, humanoid robot, which only has solid links, can move or balance through ZMP method [4-6]. Anyway, this method is only appropriate with MIMO system which has the same number of inputs and outputs. One of the other disadvantages of ZMP is that the robot moves very slowly and unflexibly. In order to improve the flexibility of robot and energy saving capabilities, some authors [7-13] put elastic components for smooth dynamic motion. By these researches [7-13], robot is flexible when the feet of robot still exist. One approach suggested $[14,15]$ is to replace the leg and foot by an elastic leg. This approach makes the robot become the underactuated MIMO system, called athlete robot (AR). The AR robot study is based on the idea of elastic legs for disabled people (Figures 1 and 2). In the case of AR, solid legs are replaced by elastic legs and two torques are substituted by one torque (Figure 3 ). Ryuma Niiyama and his colleagues studied this class of robots but their efforts were focused on the experimental results and real biomechanical structure. The dynamic equations and control algorithm were not analyzed. This paper will present the dynamic equation of AR.
Classical methods of ZMP control for humanoid robot [4-6] become useless for ARs due to structure of AR as a MIMO underactuated system. Some researches [16-18] simplified the complicated structure of two-legged robot into simple form: spring-load inverted pendulum (SLIP). Anyway, the SLIP model is not completely equivalent to the former MIMO model. Hence, the controller which is based on the former complicated model will be more reliable. Beside the opinion of control methods for MIMO underactuated nonlinear system [19], this paper also presents new idea that three proportional-controllers (P-controllers) are designed to transform AR to a SIMO system. Hence, it will be more convenient to use multiple control methods for SIMO underactuated system [20-23]. A basic method of hierarchical sliding mode (HSM) control algorithm for SIMO system, which was presented by Qian et al. [20], can balance a SIMO system. Other related works [21-23] used HSM to balance a specific SIMO system. Qian used mathematical methods to prove the stability of sliding surfaces of each layer in [20]. Despite the remarkable contribution in [20], there is still a boundary that control parameters "should" be in. Therefore, based on that boundary, genetic algorithm (GA) is used in our paper to find the appropriate control parameters. 


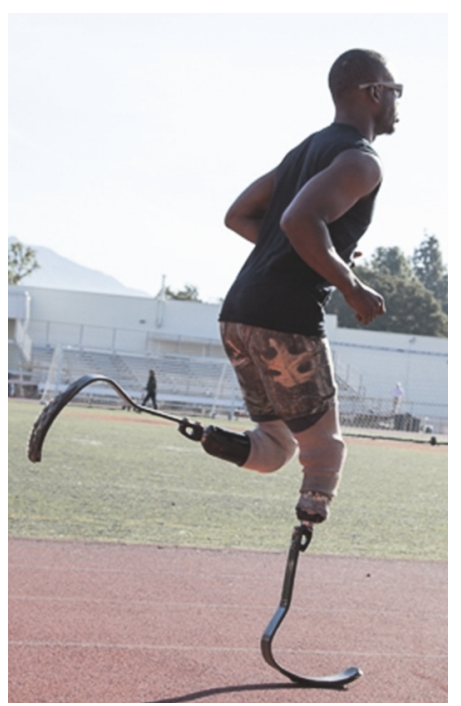

FIgURE 1: Motion of disabled people with elastic legs.

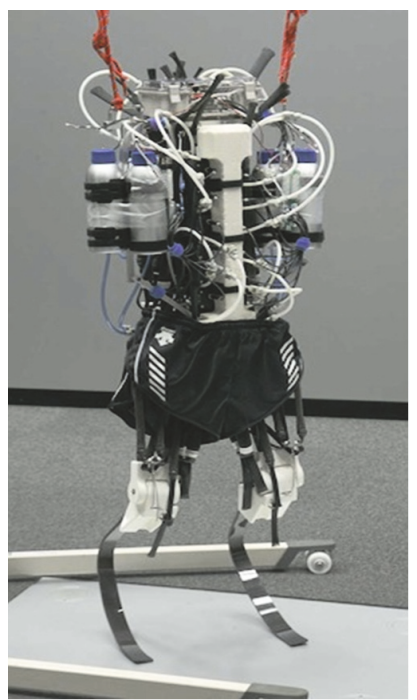

Figure 2: AR, University of Tokyo, Japan [14].

Also, GA is also used to design the prescribed trajectory of motion that defines a step of robot.

The paper concludes five sections. The dynamic equations of AR are generated in Section 2. Section 3 infers the mathematical transformation from MIMO underactuated nonlinear structure of AR into a SIMO one. Section 3 also presents application of HSM controller for that robot. Section 4 introduces simulation results. A conclusion in Section 5 ends the paper.

\section{Mathematical Model}

The elastic legs are designed to be able to self-balance when there is not external force on them (Figure 4) and to accumulate elastic energy for motion. We introduce the following notations:
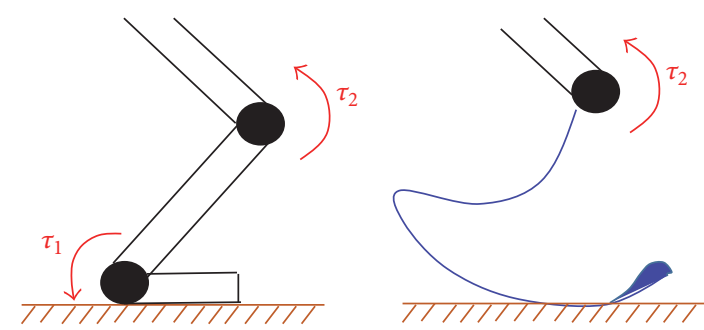

FIGURE 3: Correlation between leg of solid robot and elastic leg of AR.
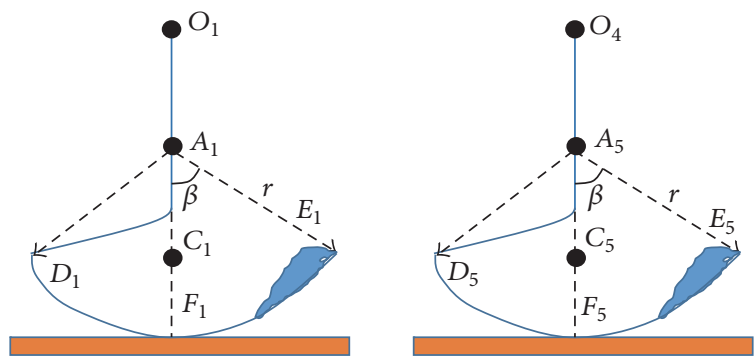

FIGURE 4: Self-balanced position of elastic legs.

(i) $C_{i}$ : mass center of link $i(i=1,5)$

(ii) $O_{i}$ : (with $\left.(i=1,2,3,4)\right)$ connecting point of link $i$ and link $i+1$

(iii) $A_{i}$ : center of the curved part of elastic leg $(i=1,5)$

(iv) $D_{i}, E_{i}$ : two-edge points of elastic legs with coordinates: $\left(x_{D i}, y_{D i}\right)$ and $\left(x_{E i}, y_{E i}\right)(i=1,5)$

(v) $F_{i}$ : middle point of elastic legs with coordinate: $\left(x_{F i}, y_{F i}\right)(i=1,5)$

(vi) $\beta=\widehat{E_{i} A_{i} C_{i}}$ : angle of curved part of elastic legs

Main parameters of AR are described in Figure 5 as follows:

(i) $m_{i}$ : mass of $i(i=1,2,3,4,5) ; m_{1}=m_{5} ; m_{2}=m_{4}(\mathrm{~kg})$

(ii) $l_{i}$ : length of link $i(i=2,3,4) ; l_{2}=l_{4} ; l_{3}=0(\mathrm{~m})$

(iii) $\alpha_{1}$ : angle between link 1 and vertical axis, obtaining $\alpha_{1}=\widehat{C_{1} O_{1} y^{\prime}}(\mathrm{rad})$

(iv) $\alpha_{i}$ : angle between next link and link $i(i=2,3,4,5)$ (rad)

(v) $I_{i}$ : inertial moment of link $i(\mathrm{kgm} 2)$

(vi) $r$ : radius of hunch part (m)

(vii) $k$ : rotational spring coefficient $(\mathrm{Nm} / \mathrm{rad})$

Behavior of elastic leg is determined by Castigliano's Theorem [24] which provides a good tool for analyzing forces on curved components. AR can be regarded as an equivalent inverted pendulum in Figure 6 where the elastic legs are equivalent to springs.

Consider Figure 6(b) that describes the equivalent model of AR's leg. The strain potential energy of system can be defined as

$$
P_{\text {strain }}=\frac{1}{2} k \alpha_{1}^{2}
$$




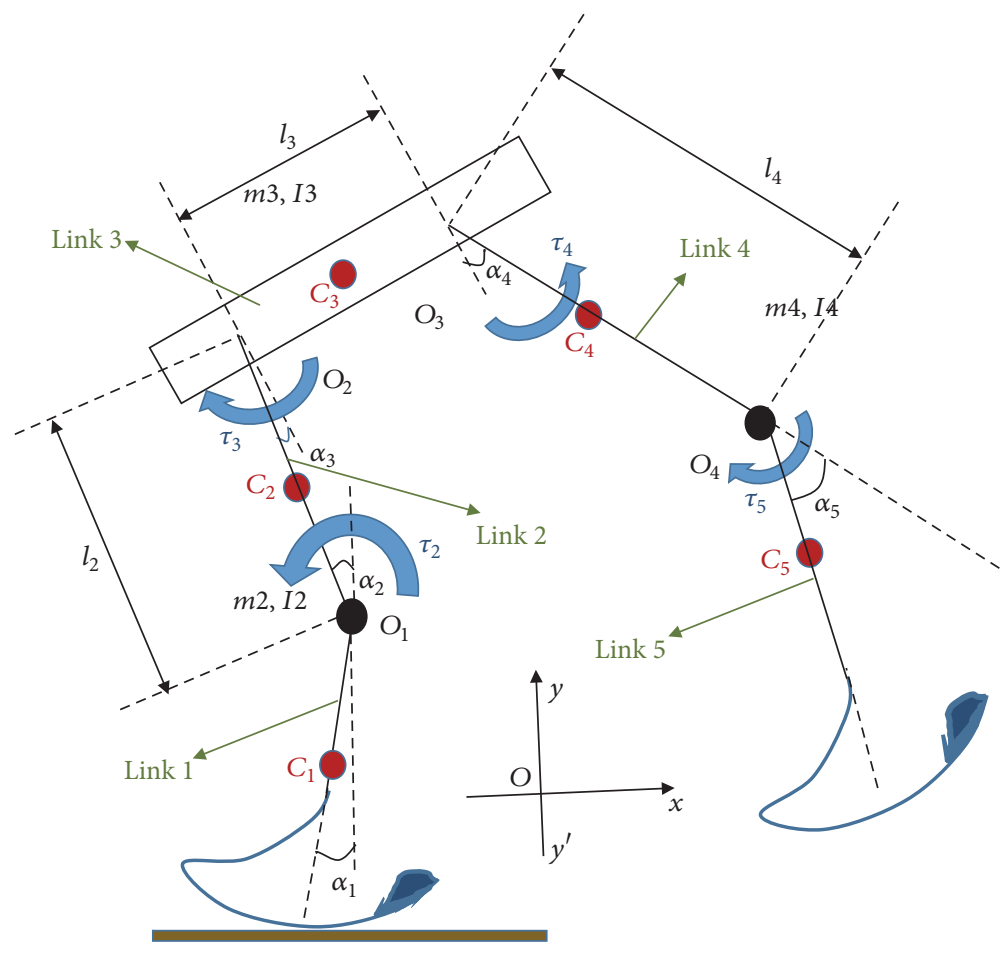

FIGURE 5: Mathematical model of AR.
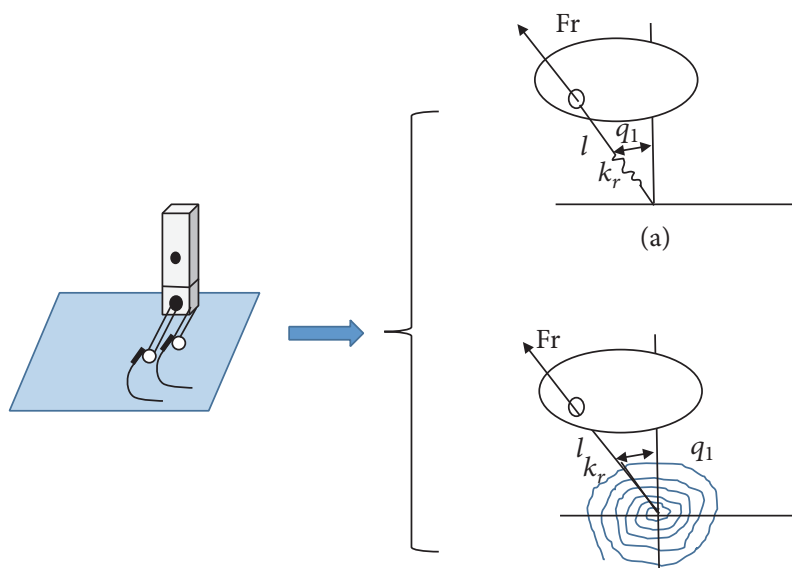

(a)

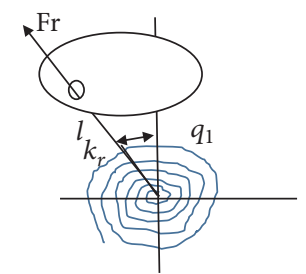

(b)

FIGURE 6: Equivalent pendulum model: (a) as linear spring and (b) as rotational spring.

Total potential energy of system is

$$
P=g \sum_{i=1}^{5} m_{i} y_{C i}+P_{\text {strain }}
$$

Kinetic energy of system is

$$
K=\sum_{i=1}^{5}\left[\frac{m_{i}}{2}\left(\dot{x}_{C i}^{2}+\dot{y}_{C i}^{2}\right)+\frac{I_{i}}{2} \dot{\alpha}_{i}^{2}\right] .
$$

Lagrange operator is

$$
L=K-P .
$$

By using Euler-Lagrange method, dynamic equations will be

$$
\begin{aligned}
& \frac{\partial L}{\partial \alpha_{i}}-\frac{d}{d t}\left(\frac{\partial L}{\partial \dot{\alpha}_{i}}\right)=\tau_{i} \quad(i=2,3, \ldots, 5) \\
& \frac{\partial L}{\partial \alpha_{1}}-\frac{d}{d t}\left(\frac{\partial L}{\partial \dot{\alpha}_{1}}\right)=0 .
\end{aligned}
$$

From (5), the dynamic model can be written as

$$
J\left(\alpha_{i}\right) \ddot{\alpha}=F\left(\alpha_{i}, \dot{\alpha}_{i}\right)+\tau,
$$

where $\alpha=\left[\begin{array}{llll}\alpha_{1} & \alpha_{2} & \cdots & \alpha_{5}\end{array}\right]^{T}, \tau=\left[\begin{array}{lllll}0 & \tau_{2} & \tau_{3} & \tau_{4} & \tau_{5}\end{array}\right]^{T}$, and the matrices $J, F$ are calculated by MATLAB/Simulink simulation. New variables $q_{i}$ are defined to simplify forms of equation (Figure 7).

Relation of variables $\alpha_{i}$ and $q_{i}$ is inferred in

$$
\begin{aligned}
& \alpha_{1}=q_{1} ; \\
& \alpha_{2}=q_{1}-q_{2} ; \\
& \alpha_{3}=q_{2}+q_{3} ; \\
& \alpha_{4}=-q_{3}-q_{4} ; \\
& \alpha_{5}=q_{5}-q_{4}
\end{aligned}
$$




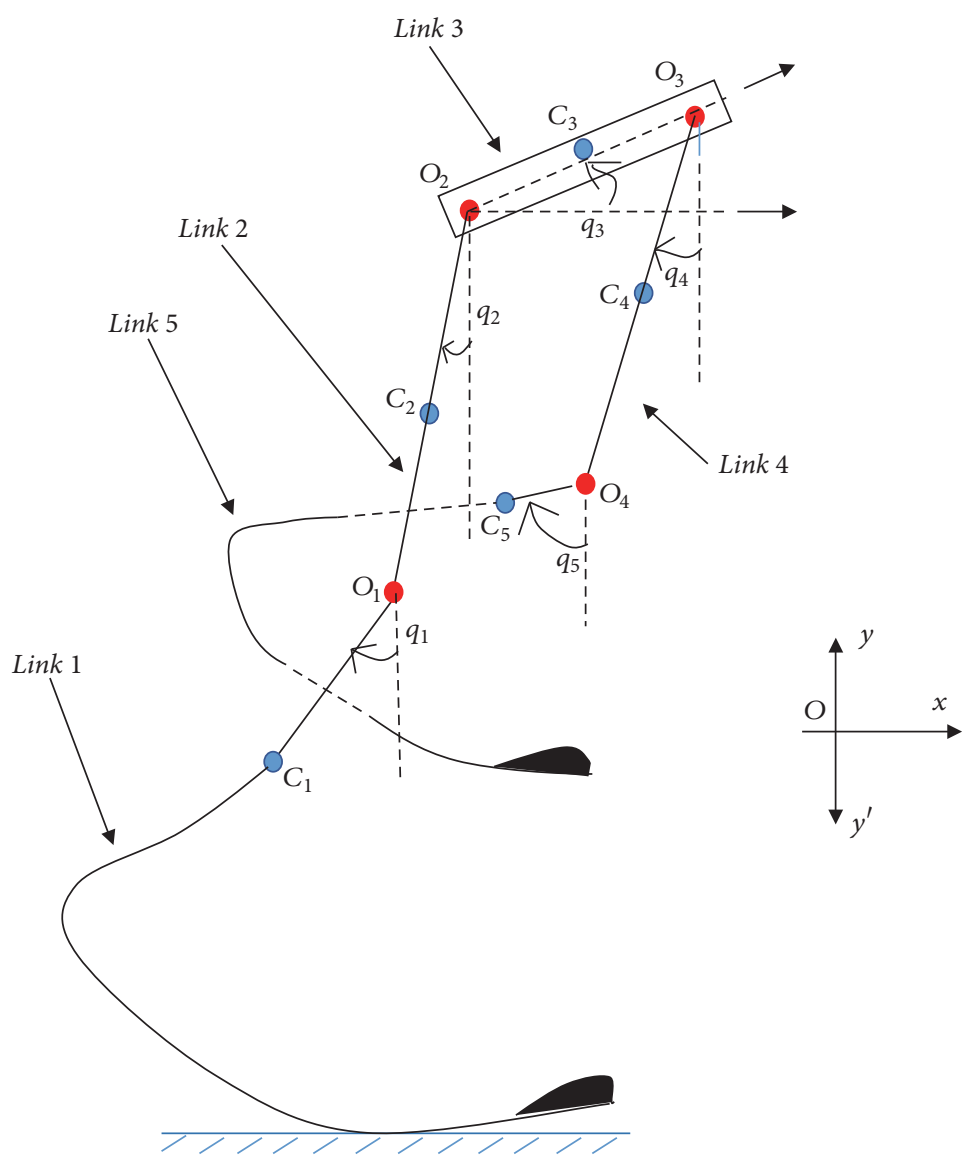

FIGURE 7: Model of AR with variables are referenced to the vertical axis.

$$
\begin{aligned}
& q_{1}=\alpha_{1} ; \\
& q_{2}=q_{1}-\alpha_{2} ; \\
& q_{3}=\alpha_{3}-q_{2} ; \\
& q_{4}=-q_{3}-\alpha_{4} ; \\
& q_{5}=q_{4}+\alpha_{5}
\end{aligned}
$$

Also, denote by $\alpha_{i d}$ and $q_{i d}$ the reference signal of variables $\alpha_{i}$ and $q_{i}$. Consider $q_{0}=$ const $>0$ the angle between one leg and the vertical axis when both legs touch the ground. Sample-time $T$ is defined as period time of a step of robot

Many authors [25-27] proposed several solutions for designing the oscillators for the motion of two-legged walking robots. Their methods are very complex and based on intuition and developed by simulations and experiments. In this paper, an algorithm is proposed based on GA.

We consider the reference trajectories of $q_{1}, q_{2}, q_{3}, q_{4}$, and $q_{5}$ as $q_{1 d}=q_{0}=$ const, $q_{2 d}=q_{0}=$ const, $q_{3 d}=0, q_{4 d}, q_{5 d}$. Reference trajectories of $q_{4}$ and $q_{5}$ are described in Figure 9.

Coordinates $\left(x_{A j}, y_{A j}\right)$ and $\left(x_{B j}, y_{B j}\right)$ have to be selected through GA.

\section{Control Algorithm}

In order to use HSC algorithms, new variables will be defined as

$$
e_{i}=\alpha_{i}-\alpha_{i d} \quad(i=1,2, \ldots, 5),
$$

where $\alpha_{d}=\left[\begin{array}{llll}\alpha_{1 d} & \alpha_{2 d} & \cdots & \alpha_{5 d}\end{array}\right]^{T}, e=\left[\begin{array}{llll}e_{1} & e_{2} & \cdots & e_{5}\end{array}\right]^{T}$.

The dynamic model (6) is rewritten as

$$
\begin{aligned}
\ddot{e}= & J^{-1} F+J^{-1}\left[\begin{array}{lllll}
0 & 0 & \tau_{3} & \tau_{4} & \tau_{5}
\end{array}\right]^{T}-\ddot{\alpha}_{d} \\
& +J^{-1}\left[\begin{array}{lllll}
0 & \tau_{2} & 0 & 0 & 0
\end{array}\right]^{T} .
\end{aligned}
$$

A simply proportional controller for links 3, 4, and 5 is proposed

$$
\tau_{i}=K_{p i} e_{i} \quad(i=3,4,5)
$$

Also, define

$$
\begin{aligned}
f & =\left[\begin{array}{llll}
f_{1} & f_{2} & \cdots & f_{5}
\end{array}\right]^{T} \\
& =J^{-1} F+J^{-1}\left[\begin{array}{lllll}
0 & 0 & \tau_{3} & \tau_{4} & \tau_{5}
\end{array}\right]^{T}-\ddot{\alpha}_{d}, \\
b & =\left[\begin{array}{llll}
b_{1} & b_{2} & \cdots & b_{5}
\end{array}\right]^{T},
\end{aligned}
$$

where $b$ is the second column of matrix $J^{-1}$. 
Substitute (10) and (11) into (9), and obtain

$$
\ddot{e}=f+b \tau_{2} .
$$

By using the conventional change of control inputs (10), the dynamic model (6) is transformed in SIMO models in (12). The AR is a five-order system and it is impossible to calculate directly $J^{-1}$ with just unknown variables due to its complexity and limitation of simulating software (MATLAB/Simulink) (only 25000 characters can appear on MATLAB window). Hence, exact dynamic equations cannot be described visibly. Anyway, simulation process has to be implemented following Figure 10.

From (8) to (12), the error model will be

$$
\ddot{e}_{i}=f_{i}+b_{i} \tau_{2} \quad(i=1,2, \ldots, 5) .
$$

Equation (13) has the form of equation of a SIMO system. Therefore, a solution of using controller which is suitable for high-order SIMO system can be considered. This controller stabilizes variables $e_{i} \stackrel{t \rightarrow \infty}{\longrightarrow} 0$. This leads to $\alpha_{i} \stackrel{t \rightarrow \infty}{\longrightarrow} \alpha_{i d}$.

Hierarchical sliding mode of structure of hierarchical sliding surfaces is shown in Figure 10.

From the description in Figure 11, sliding surfaces are chosen

$$
\begin{aligned}
& s_{i}=c_{i} e_{i}+\dot{e}_{i} \quad(i=1,2, \ldots, 5) \\
& S_{i}=a_{i-1} S_{i-1}+s_{i} \quad(i=1,2, \ldots, 5)
\end{aligned}
$$

where $a_{i-1}=$ const and $a_{0}=S_{0}=0$.

$$
S_{i}=\sum_{r=1}^{i}\left(\prod_{j=r}^{i} a_{j}\right) s_{r} \quad(i=1,2, \ldots, 5) .
$$

Derivative (16) with respect to time yields

$$
\dot{S}_{i}=\sum_{r=1}^{i}\left(\prod_{j=r}^{i} a_{j}\right) \dot{s}_{r} \quad(i=1,2, \ldots, 5) .
$$

From (14) and (15), we deduce that the $i$ th layer SMC comprises the information of $(i-1)$ th, $(i-2)$ th, $\ldots, 1$ st layer, and subsystem layer. Hence, define the ith layer sliding mode control law as

$$
u_{i}=u_{i-1}+u_{\mathrm{eq} i}+u_{\mathrm{sw} i} \quad(i=1,2, \ldots, 5) .
$$

Here, $\tau_{0}=0$. And $u_{\text {swi }}, u_{\text {eqi }}$ is switching and equivalent control law for $i$ th layer. Let

$$
u_{\mathrm{eq} i}=-\frac{c_{i} \dot{e}_{i}+f_{i}}{b_{i}} \quad(i=1,2, \ldots, 5)
$$

Define Lyapunov function for $i$ th layer as

$$
V_{i}=\frac{S_{i}^{2}}{2} \quad(i=1,2, \ldots, 5)
$$

Derive (20), and from (17), we obtain

$$
\left(\dot{V}_{i}=\right) S_{i} \dot{S}_{i}=S_{i}\left[\sum_{r=1}^{5}\left(\prod_{j=r}^{5} a_{j}\right) \dot{s}_{r}\right] \quad(i=1,2, \ldots, 5) .
$$

Derive (14), and substitute (14) and (18) into new results which is obtained by deriving (14), which yields

$$
\begin{aligned}
\dot{S}_{i}= & \sum_{l=1}^{i}\left[\sum_{\substack{r=1 \\
r \neq l}}^{i}\left(\prod_{j=r}^{i} a_{j}\right) b_{r}\right] u_{\mathrm{eql}} \\
& +\sum_{l=1}^{i}\left[\sum_{r=1}^{i}\left(\prod_{j=r}^{i} a_{j}\right) b_{r}\right] u_{\mathrm{swl}} \quad(i=1,2, \ldots, 5) .
\end{aligned}
$$

By considering stability of $i$ th layer sliding surface, let

$$
\dot{S}_{i}=-k_{i} S_{i}-\eta_{i} \operatorname{sgn} S_{i} \quad\left(\text { where } k_{i}>0, \eta_{i}>0\right)
$$

From (22) and (23), switching control law of $i$ th layer can be obtained as

$$
\begin{aligned}
& u_{\mathrm{sw} i}=-\sum_{l=1}^{i-1} u_{\mathrm{sw} l} \\
&-\frac{\left[\sum_{l=1}^{i=1}\left[\sum_{r=1, r \neq l}^{i}\left(\prod_{j=r}^{i} a_{j}\right) b_{r}\right] u_{\mathrm{eql}}-\left(k_{i} S_{i}+\eta_{i} \operatorname{sgn} S_{i}\right)\right]}{\sum_{r=1}^{i}\left(\prod_{j=r}^{i} a_{j}\right) b_{r}} \\
&(i=1,2, \ldots, 5),
\end{aligned}
$$

and the final control law for controller is selected as

$$
\begin{aligned}
\tau_{2} & =u_{5} \\
& =\frac{\left[\sum_{r=1}^{5}\left(\prod_{j=r}^{5} a_{j}\right) b_{r} u_{\mathrm{eq} r}-\left(k_{5} S_{5}+\eta_{5} \operatorname{sgn} S_{5}\right)\right]}{\sum_{r=1}^{5}\left(\prod_{j=r}^{5} a_{j}\right) b_{r}} .
\end{aligned}
$$

From general results in [20], we obtain Theorems 1 and 2 below.

Theorem 1. Consider that equations of error of system (9) are described in (13). If control law is chosen as (25) and ith sliding surfaces are identified as in (16), then $S_{i}$ is asymptotically stabilized.

Proof. The Lyapunov function of $i$ th layer is chosen as in (20). From (23), we obtain

$$
\dot{V}_{i}=S_{i} \dot{S}_{i}=S_{i}\left(-k_{i} S_{i}-\eta_{i} \operatorname{sgn} S_{i}\right) \quad(i=1,2, \ldots, 5) .
$$

Integrating two sides of formula (21), we obtain

$$
\int_{0}^{t} \dot{V}_{i} d \tau=\int_{0}^{t}\left(-k_{i} S_{i}^{2}-\eta_{i}\left|S_{i}\right|\right) d \tau \quad(i=1,2, \ldots, 5) .
$$

Consider

$$
\begin{aligned}
V_{i}(0) & =V_{i}(t)+\int_{0}^{t}\left(k_{i} S_{i}^{2}+\eta_{i}\left|S_{i}\right|\right) d \tau \\
& \geq \int_{0}^{t}\left(k_{i} S_{i}^{2}+\eta_{i}\left|S_{i}\right|\right) d \tau \quad(i=1,2, \ldots, 5) .
\end{aligned}
$$

Therefore, we obtain

$$
\lim _{t \rightarrow \infty} \int_{0}^{t}\left(k_{i} S_{i}^{2}+\eta_{i}\left|S_{i}\right|\right) d \tau \leq V_{i}(0)<\infty
$$

$$
(i=1,2, \ldots, 5) \text {. }
$$




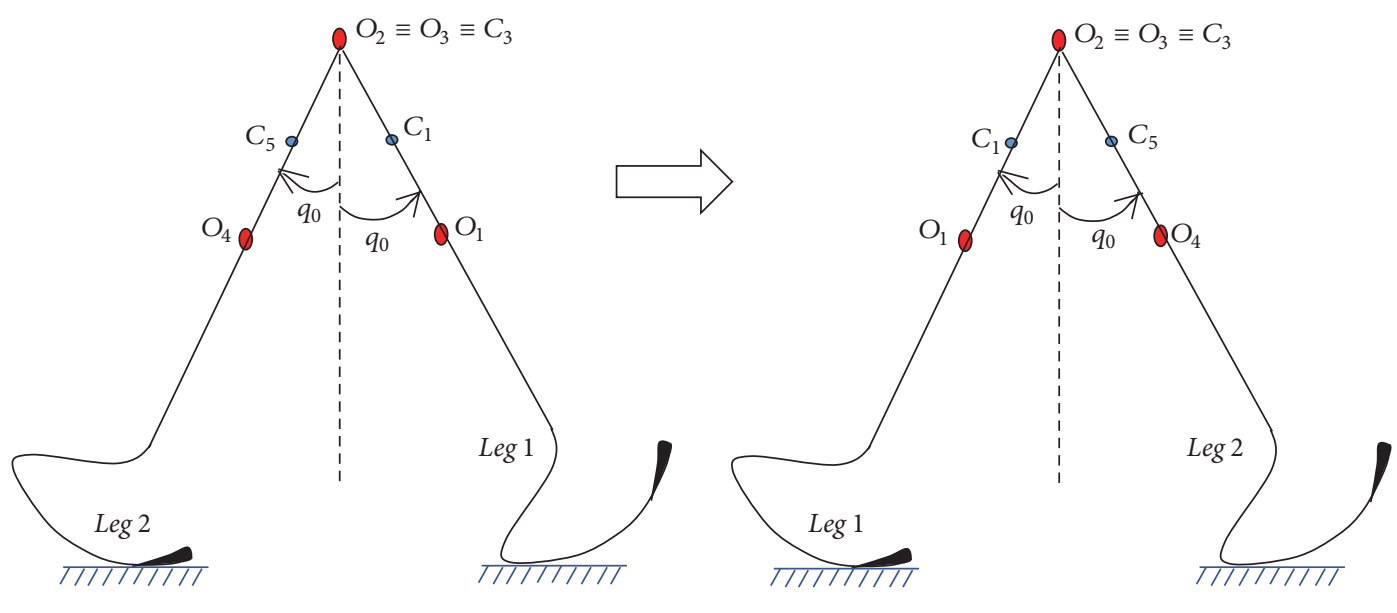

(a)

(b)

Figure 8: Position of two legs. (a) Before the step and (b) after the step.

According to Barbalat lemma, there exists

$$
\begin{aligned}
\lim _{t \rightarrow \infty}\left(k_{i} S_{i}^{2}+\eta_{i}\left|S_{i}\right|\right) d \tau \leq V_{i}(0)<\infty & \\
& (i=1,2, \ldots, 5) .
\end{aligned}
$$

From (30), it means that $\lim _{t \rightarrow \infty} S_{i}=0$. Hence, the $i$ th sliding surface of $S_{i}$ is asymptotically stable.

Theorem 2. Consider that equations of error of system (9) are described in (13). If control law is chosen as (25) and ith subsystem sliding surfaces are identified as in (14), then $s_{i}$ is asymptotically stabilized.

Proof. Assume $s_{i}$ is not asymptotically stabilized when conditions in Theorem 2 are satisfied

$$
\lim _{t \rightarrow \infty} s_{i} \neq 0 \quad(i=1,2, \ldots, 5) .
$$

Hence, from (16), we obtain

$$
\begin{aligned}
\lim _{t \rightarrow \infty} S_{i} & =\lim _{t \rightarrow \infty} \sum_{r=1}^{i}\left(\prod_{j=r}^{i} a_{j}\right) s_{r}=\sum_{r=1}^{i}\left(\prod_{j=r}^{i} a_{j}\right) \lim _{t \rightarrow \infty} s_{r} \\
& \neq 0 \quad(i=1,2, \ldots, 5) .
\end{aligned}
$$

Equation (32) contradicts Theorem 1. Therefore, we obtain

$$
\lim _{t \rightarrow \infty} s_{i}=0 \quad(i=1,2, \ldots, 5)
$$

and $s_{i}$ is asymptotically stabilized.

Theorems 1 and 2 prove the stability of sliding surface. However, the stability of $e_{i}$ is not guaranteed. Therefore, searching algorithm, such as genetic algorithm (GA), is a solution to find appropriate control parameters in (25). In this case, GA is defined by population of 40 individuals. Each individual contains 24 chromosomes which include values of the following: (i) Control parameters: $a_{1}, a_{2}, a_{3}, a_{4}, a_{5}, c_{1}, c_{2}, c_{3}, c_{4}, c_{5}$, $\eta_{5}, k_{5}, K_{p 1}, K_{p 2}, K_{p 3}$

(ii) Trajectory parameters: $x_{A 4}, x_{A 5}, x_{B 4}, x_{B 5}, y_{A 4}, y_{A 5}$, $y_{B 4}, y_{B 5}$

(iii) Half-period time of a motion cycle (time for a step of AR): $T$ (if AR moves fast, then $T$ is chosen in the range: $0(\mathrm{~s}) \leq T \leq 2(\mathrm{~s}))$

The individuals chosen for crossover process have to satisfy the following conditions:

(i) All $y_{D i}, y_{E i}$, and $y_{F i}(i=1,5)$ are positive for $t=0 \rightarrow$ $T$.

(ii) $0 \leq x_{A i} \leq x_{B i} \leq T(i=4,5)$.

Fitness function of GA is defined as

$$
J=\frac{1}{T} \sum_{i=1}^{5} \zeta_{i}^{2} \quad(i=1,2, \ldots, 5),
$$

where $\zeta_{i}=q_{i}-q_{d i}$.

In GA process, the fitness function of GA is designed to evaluate the results after each loop of searching. If each link tracks trajectory, then $\zeta_{i} \rightarrow 0$ and $\sum_{i=1}^{5} \zeta_{i}^{2} \rightarrow 0$. However, sample time of step $T$ is also chosen through GA. Only $\sum_{i=1}^{5} \zeta_{i}^{2}$ cannot represent the ability of tracking trajectory in a period of time. Therefore, fitness function should be selected as $J=$ $(1 / T) \sum_{i=1}^{5} \zeta_{i}^{2}$. The detected result which generates smaller $J$ from (34) will be considered as better.

The GA process is shown in Figure 12.

In Figure 12, firstly, 40 random couples were created. In each individual in couples, the structure of gene concludes encoded parameters of control system and reference trajectories (control parameters: $a_{1}, a_{2}, a_{3}, a_{4}, a_{5}, c_{1}, c_{2}, c_{3}, c_{4}, c_{5}, \eta_{5}$, $\left.k_{5}, K_{p 1}, K_{p 2}, K_{p 3}, x_{A 4}, x_{A 5}, x_{B 4}, x_{B 5}, y_{A 4}, y_{A 5}, y_{B 4}, y_{B 5}\right)$. We denote a constant value $J_{\min }$ to check if the result is best or not. $J_{\min }$ will be updated if there is better value and the result that makes $J<J_{\min }$ will be stored as the best result. After 


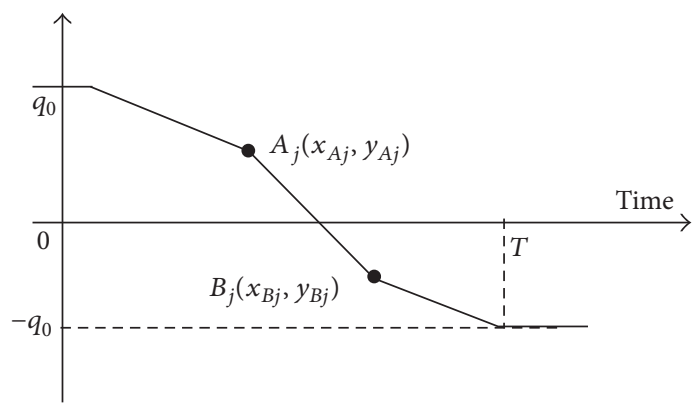

FIGURE 9: Reference trajectories $q_{j d}(j=4,5)$.

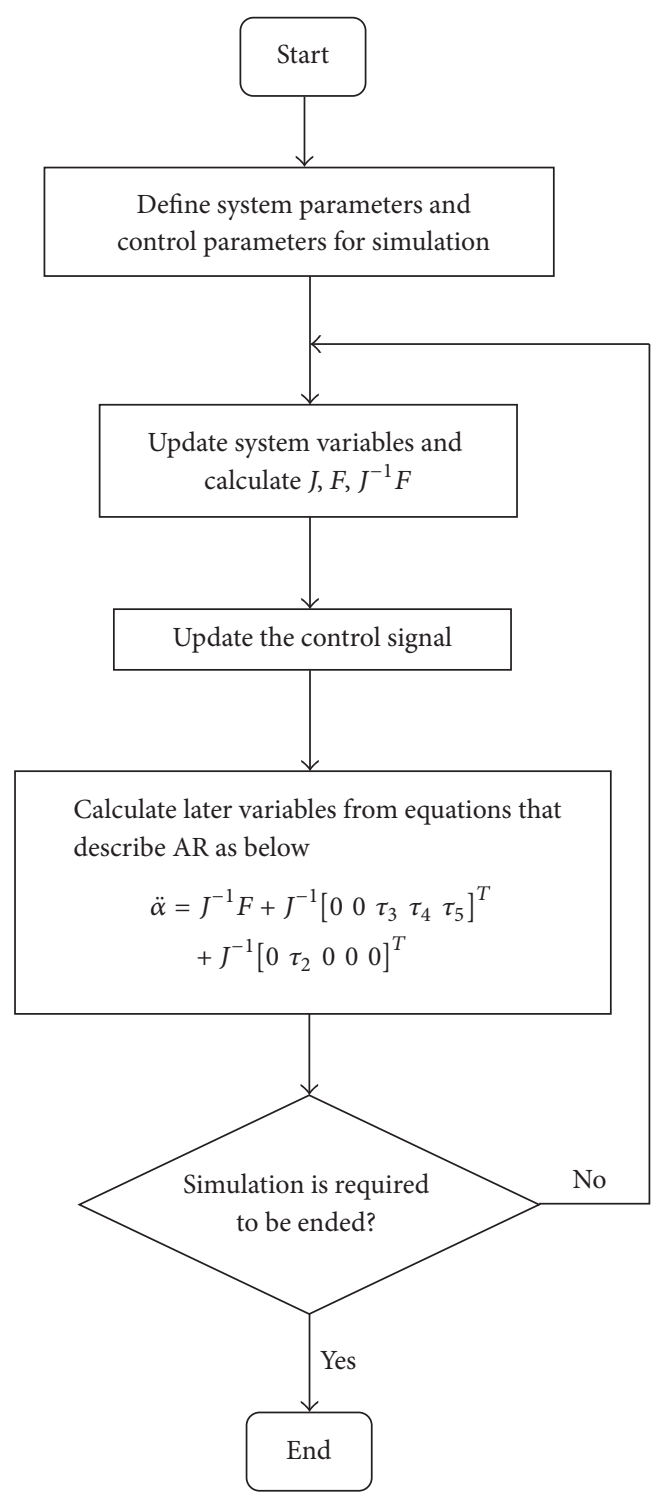

FIGURE 10: Simulation process. 


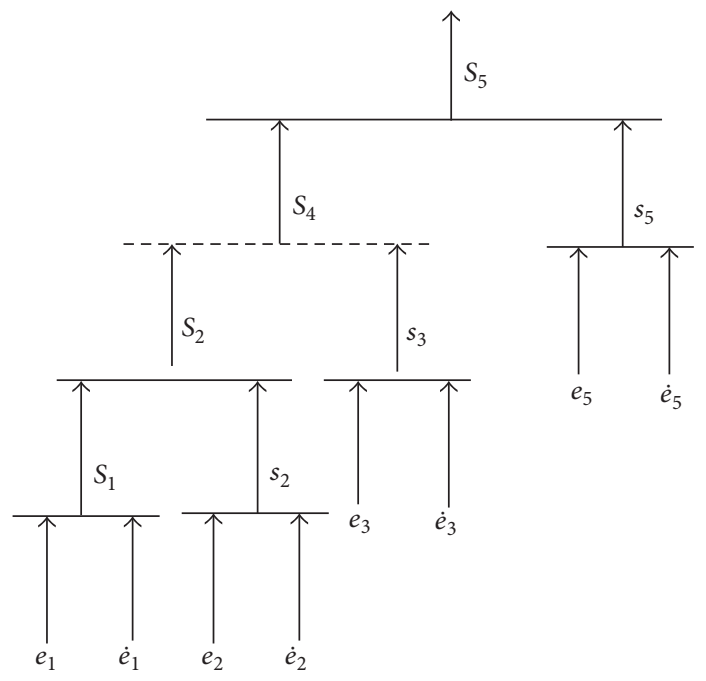

FIGURE 11: Hierarchical sliding surfaces structure for system which has one control input and five output variables.

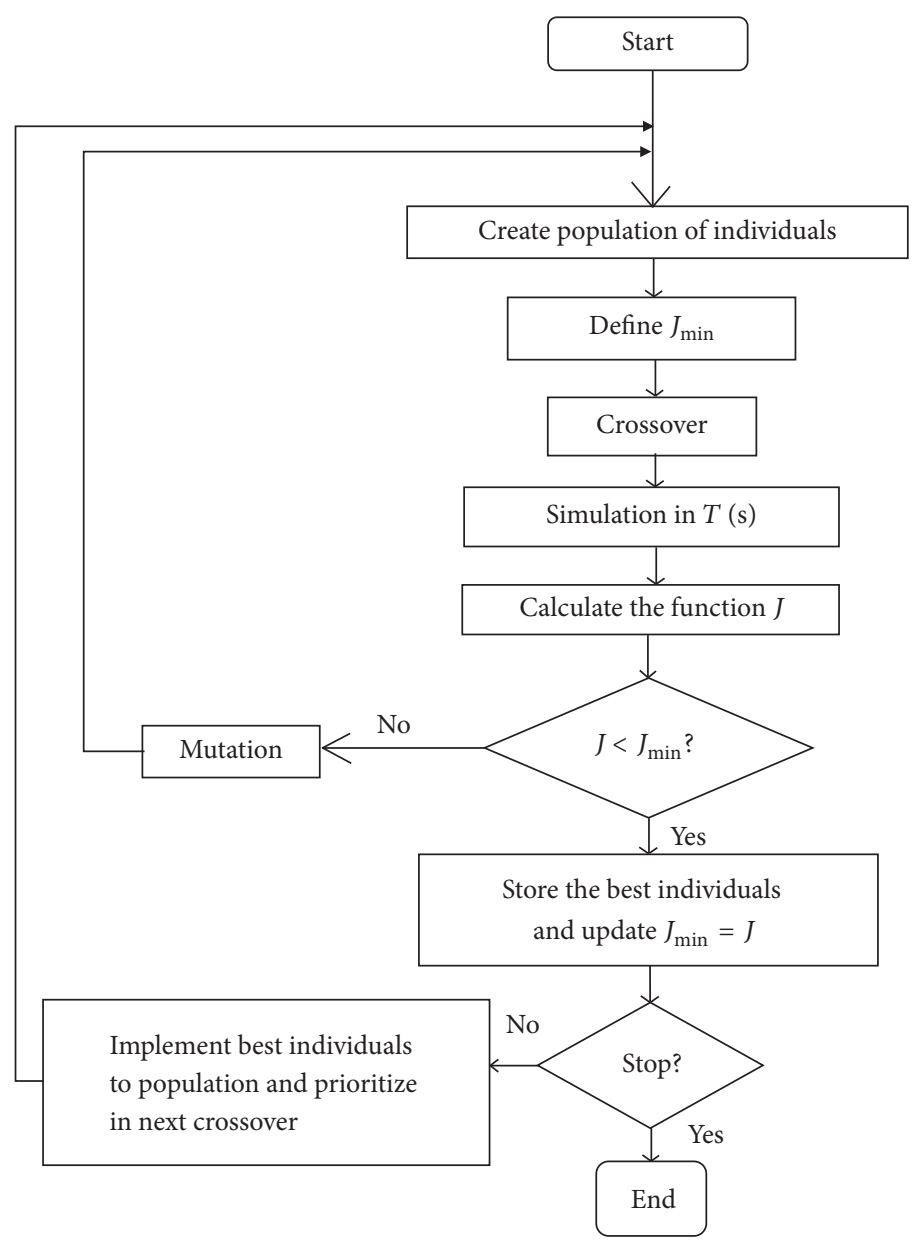

FIGURE 12: Flowchart of genetic calculation process. 


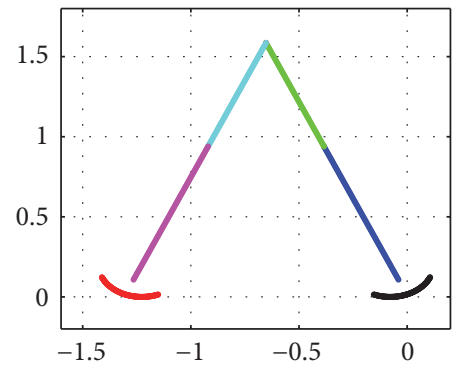

(a)

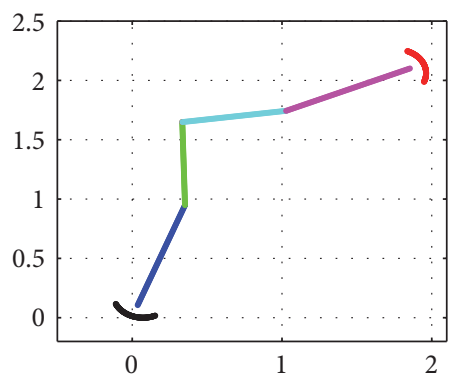

(b)

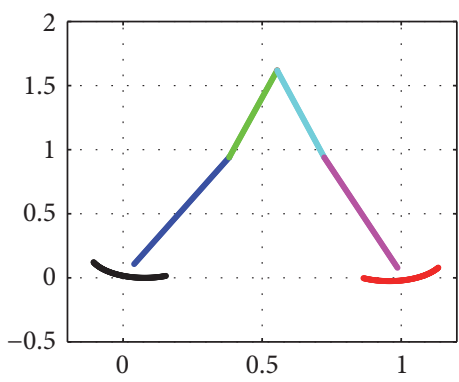

(c)

Figure 13: AR at representative time (a) $t=0$ (s), (b) $t=T=1.01$ (s), and (c) $t=1.12$ (s). Number 1; curved part of elastic part of leg 2; number 2: linear part of elastic part of leg 2; number 3: link 4; number 4: link 2; number 5: linear part of elastic part of leg 1; number 6: curved part of elastic part of leg 1 .

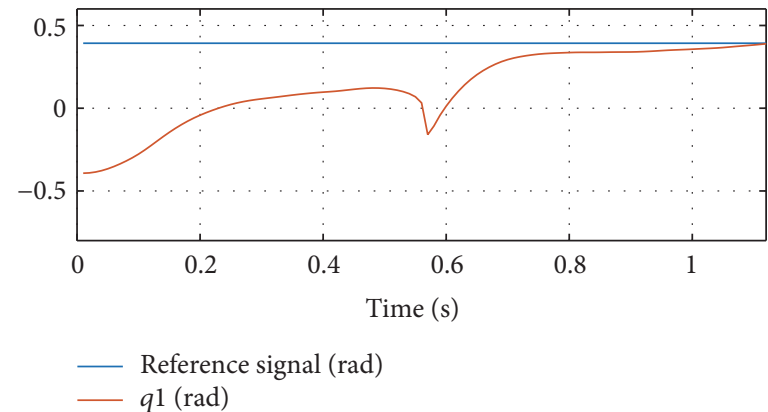

FIGURE 14: Trajectory of $q_{1}(\mathrm{rad})$.

crossover couples, 80 new individuals are created. By checking the function $J$ of each individual after each simulation, better individuals have more priority to become couples and have more offspring in next generation. Worse individuals have less priority to give their gene to next generation. The loop will continue until we want to stop the program.

\section{Simulation Results}

System parameters are selected as follows:

$$
\begin{aligned}
& \gamma_{1}=0.1(\mathrm{~m}) ; \gamma_{2}=1(\mathrm{~m}) ; r=0.2(\mathrm{~m}) ; \gamma_{3}=r-\gamma_{1} ; \gamma_{4}= \\
& \gamma_{2}=r ; m_{1}=0.01(\mathrm{~kg}) ; m_{2}=0.1(\mathrm{~kg}) ; m_{3}=0.5(\mathrm{~kg}) \\
& I_{1}=0.001\left(\mathrm{kgm}^{2}\right) ; I_{2}=0.02\left(\mathrm{kgm}^{2}\right) ; I_{3}=0.1\left(\mathrm{kgm}^{2}\right) ; \\
& l_{3}=0(\mathrm{~m}) ; l_{2}=0.7(\mathrm{~m}) ; g=9.81\left(\mathrm{~N} / \mathrm{kgm}^{2}\right) ; k= \\
& 0.2\left(\mathrm{~N} / \mathrm{kgm}^{2}\right) ; T=1.01(\mathrm{~s}) ; q_{0}=\pi / 8(\mathrm{rad}) .
\end{aligned}
$$

Parameters of HSM controller in (25) are found by GA method as follows:

$$
\begin{aligned}
& a_{1}=9.4 ; a_{2}=6.94 ; a_{3}=4.77 ; a_{4}=1.67 ; a_{5}=1.37 \\
& c_{1}=6.9 ; c_{2}=9.43 ; c_{3}=3.02 ; c_{4}=9.94 ; c_{5}=4.18 ; \eta_{5}= \\
& 6.88 ; k_{5}=9.83 ; K_{p 1}=76.5 ; K_{p 2}=43.3 ; K_{p 3}=81.4
\end{aligned}
$$

Parameters of reference trajectories in Figure 9 are determined as follows:

$$
\begin{aligned}
& x_{A 4}=0.1213(\mathrm{~m}) ; y_{A 4}=-0.3456(\mathrm{rad}) ; x_{B 4}=0.2526 \\
& (\mathrm{~m}) ; y_{B 4}=-0.5341(\mathrm{rad}) ; x_{A 5}=0.3536(\mathrm{~m}) ; y_{A 5}= \\
& 1.5080(\mathrm{rad}) ; x_{B 5}=0.9394(\mathrm{~m}) ; y_{B 5}=-1.4923(\mathrm{rad}) .
\end{aligned}
$$

Initial values of variables at $t=0(\mathrm{~s})$ are specified in Figure 8. Motions of AR under HSM controller are described in Figures 13 and 19.

From initial position (Figure 13(a)), after a period time $T$, AR moves to a new position (Figure 13(b)). An additional time $t_{\mathrm{add}}=0.11(\mathrm{~s})$ is needed to achieve the exact position of a final sequence. This additional time can be improved by GA techniques. Period time of a step which is $T$ will be $T+$ $t_{\text {add }}$. Trajectories of each link variables $q_{i}$ are shown in Figures $14-18$.

The walking motion of AR for a single step is shown in Figure 19.

From Figures 14 to 18, after $1.12 \mathrm{~s}$, AR finishes a step. However, in the operating process, $q_{i}$ does not track well the reference trajectories but the final positions are still close to the reference trajectories. Figures 13 and 19 also consolidate that reasoning. The motion of each link affects others significantly. Only motion of link 5 is less affected (Figure 24). Because links 3, 4, and 5 are controlled directly by P-controllers for each link (from (10)), they follow trajectories (Figures 22-24) more closely than link 1 or link 2 does. Anyway, the vibration exists in these figures due to the effect from other links. The response of link 1 and link 2 is not good 


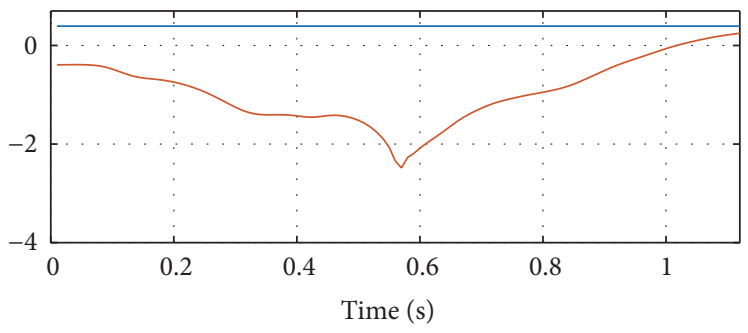

Reference signal (rad) $q 2(\mathrm{rad})$

FIgURE 15: Trajectory of $q_{2}$ (rad).

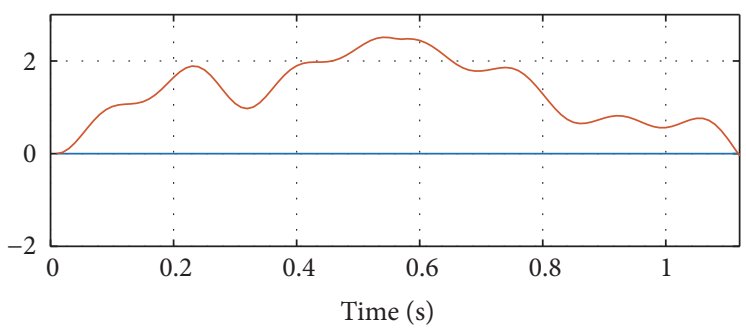

_ Reference signal (rad)

- q3 $^{(\mathrm{rad})}$

Figure 16: Trajectory of $q_{3}$ (rad).

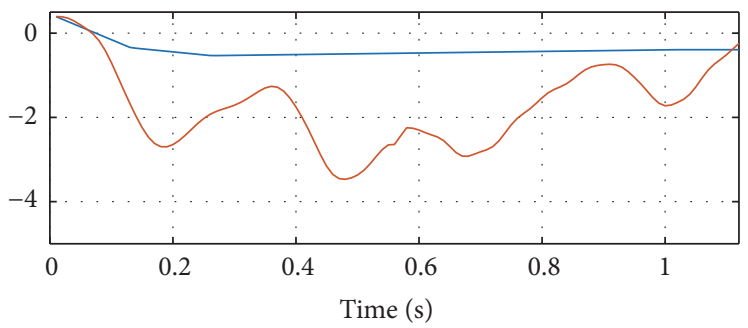

Reference signal (rad) $q 4(\mathrm{rad})$

FIGURE 17: Trajectory of $q_{4}$ (rad).

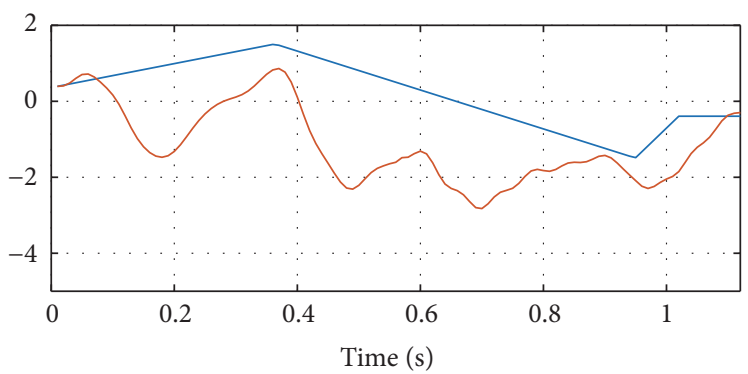

Reference signal (rad) $q 5$ ( $\mathrm{rad})$

FIGURE 18: Trajectory of $q_{5}$ (rad). 


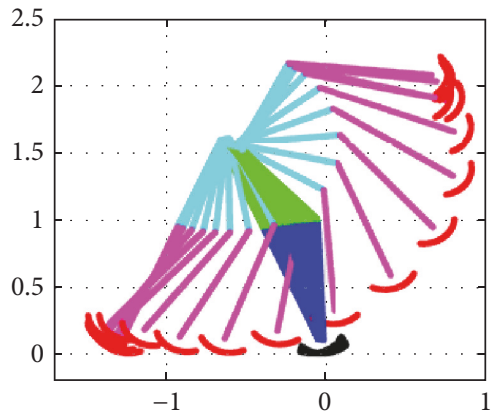

(a)

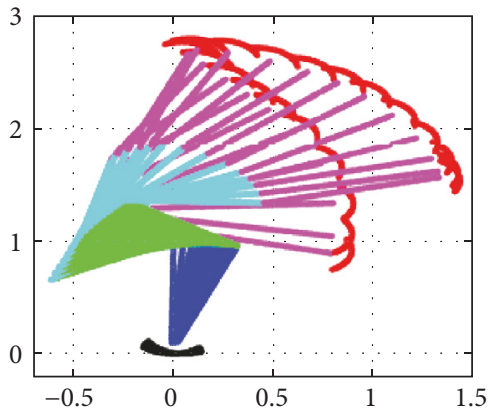

(d)

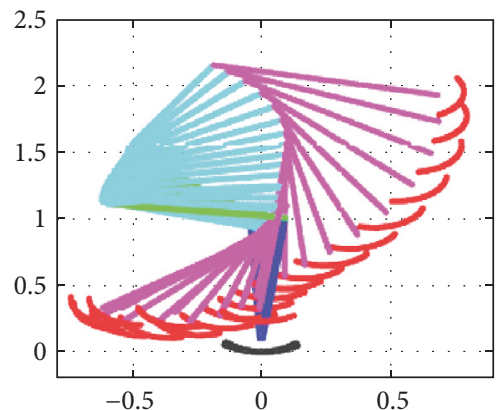

(b)

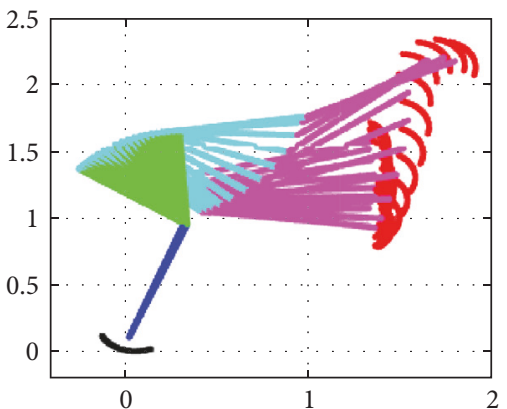

(e)

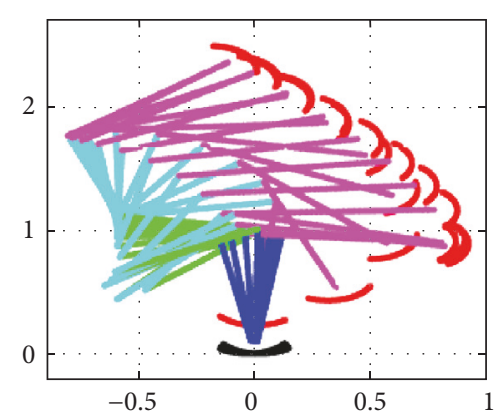

(c)

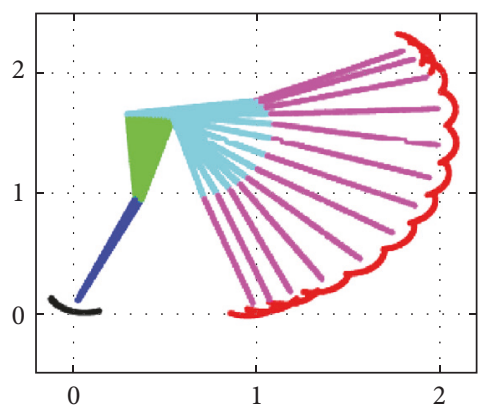

(f)

Figure 19: Motion description of AR. (a) From 0 s to 0.2 s. (b) From 0.2 s to 0.4 s. (c) From 0.4 s to 0.6 s. (d) From 0.6 s to 0.8 s. (e) From 0.8 s to $1 \mathrm{~s}$. (f) From $1 \mathrm{~s}$ to $1.12 \mathrm{~s}$.

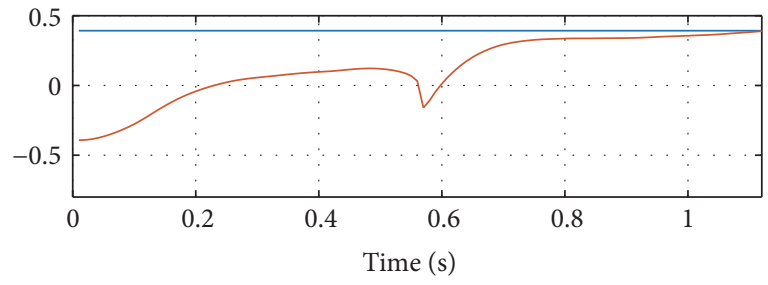

Reference signal (rad)

— Alpha 1 (rad)

FIGURE 20: Trajectory of $\alpha_{1}(\mathrm{rad})$.

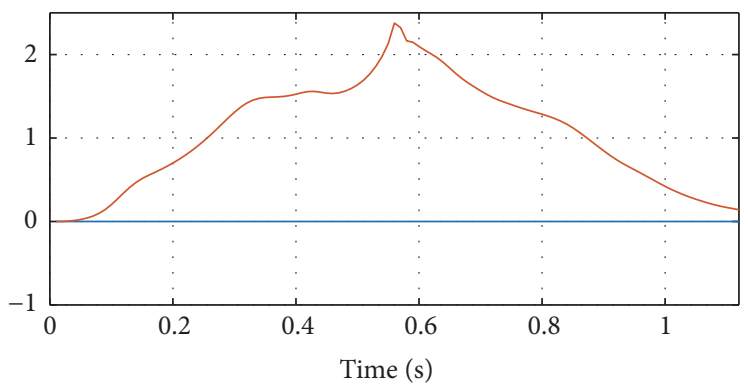

Reference signal (rad) Alpha 2 (rad)

FIgURE 21: Trajectory of $\alpha_{2}$ (rad). 


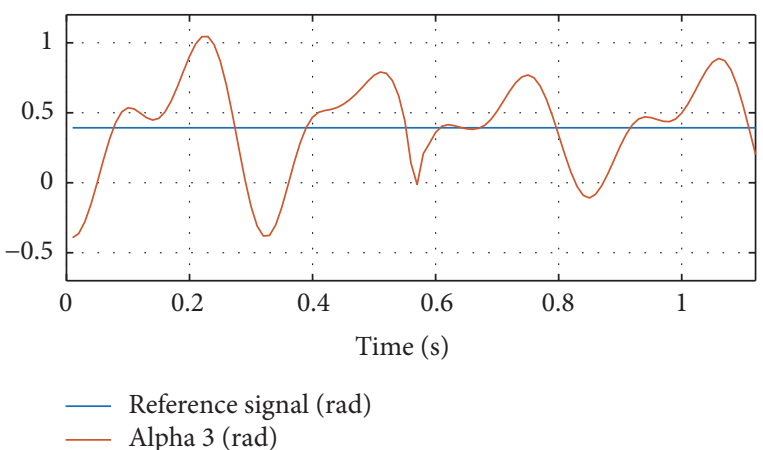

Figure 22: Trajectory of $\alpha_{3}$ (rad).

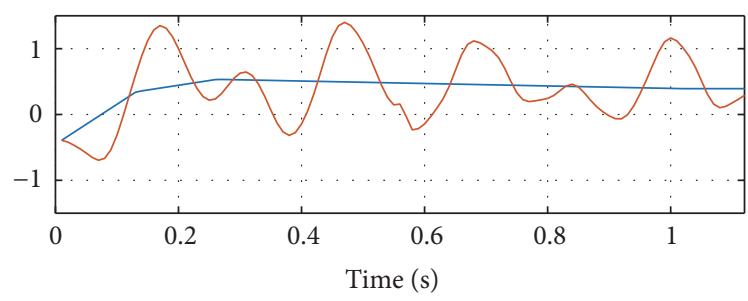

— Reference signal (rad)

- Alpha 4 (rad)

FIGURE 23: Trajectory of $\alpha_{4}$ (rad).

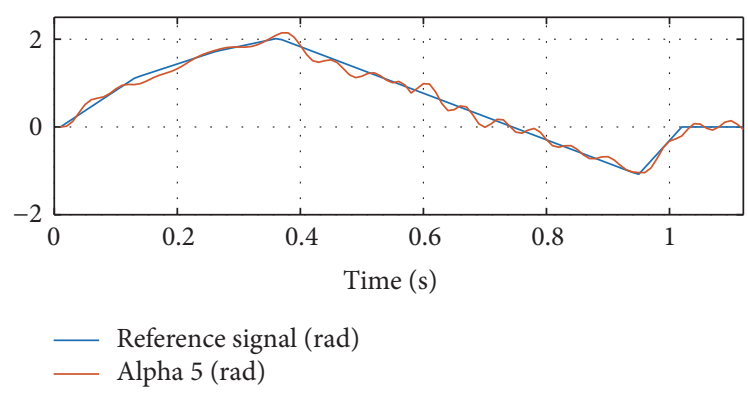

FIgURE 24: Trajectory of $\alpha_{5}(\mathrm{rad})$.

at first but HSM signal finally leads links 1 and 2 to reference trajectories at the end of period of the step (Figures 20 and 21).

\section{Conclusion}

In the paper, the authors represent method of generating dynamic equations of AR with elastic legs through EulerLagrange. Due to the complexity of structure, dynamic equations cannot be visibly shown. But, simulation can still be implemented after each loop of simulation by general matrix form of AR. A method of using three P-controllers is introduced to transform MIMO nonlinear underactuated form of AR into a SIMO system. Then, the authors also propose HSM controller, which was well implemented for SIMO system, for motion of AR in one-step period. Although sliding surfaces in that method were proved to work well, a mathematical problem is not completely guaranteed. This led to difficulty in choosing exactly control parameters. GA is proposed to solve that problem. Along with selecting the acceptable control parameters, GA is also successfully used to design the reference trajectories for motion of each link. The success of this approach in control AR robot is consolidated through simulation.

\section{Conflicts of Interest}

The authors declare that there are no conflicts of interest regarding the publication of this paper.

\section{References}

[1] V. V. Beletzky, "Nonlinear Effects in Dynamics of Controlled Two-legged Walking," in Proceedings of the Nonlinear Dynamics in Engineering Systems, International Union of Theoretical and Applied Mechanics, pp. 17-26, Springer, 1990.

[2] H. Qiang, Y. Kazuhito, K. Shuuji et al., "Planning Walking Patterns for a Biped Robot," IEEE Transactions on Robotics and Automation, vol. 17, no. 3, pp. 280-289, 2001.

[3] W. Sujan, B. Amin, S. Nathan, and S. Madhavan, "Bipedal Walking-A Developmental Design," in Proceedings of the International Symposium on Robotics and Intelligent Sensors, Procedia 41, pp. 1016-1021, Elsevier, 2012.

[4] L. Qinghua, A. Takanishi, and I. Kato, "A Biped Walking Robot having a ZMP Measurement System using Universal Forcemoment Sensors," in Proceedings of the Intelligent Robots and System, pp. 1568-1573, IEEE, 1991.

[5] S. N. Napoleon and S. Mitsuji, "Balance control analysis of humanoid robot based on ZMP feedback control," in Proceedings of the 2002 IEEE/RSJ International Conference on Intelligent Robots and Systems, pp. 2437-2442, IEEE, October 2002.

[6] D. W. Kim, N.-H. Kim, and G.-T. Park, "ZMP based neural network inspired humanoid robot control," Nonlinear Dynamics, vol. 67, no. 1, pp. 793-806, 2012.

[7] J. A. Smith and A. Seyfarth, "Elastic Leg Function in a Bipedal Walking Robot," Journal of Biomechanics, Page, vol. 40, Supplement 2, p. S306, 2007.

[8] F. Iida, Y. Minekawa, J. Rummel, and A. Seyfarth, "Toward a human-like biped robot with compliant legs," Robotics and Autonomous Systems, vol. 57, no. 2, pp. 139-144, 2009.

[9] C. K. Toh, M. Xie, H. Yang, G. Zhang, Q. P. Bui, and B. Tian, "Flexible Foot Design for Biped Walking on Uneven Terrain<," in Proceeding of International Conference on Intelligent Robotics and Applications (ICIRA 2010), Part of the Lecture Notes in Computer Science book series, vol. 6424, pp. 442-452, Springer, 2010.

[10] B.-H. Kim, "Work analysis of compliant leg mechanisms for bipedal walking robots," International Journal of Advanced Robotic Systems, vol. 10, article no. 334, 2013.

[11] A. Ming, K. Sato, R. Sato, E. Kazama, I. Miyamoto, and M. Shimojo, "Development of robot leg composed of parallel linkage and elastic spring for dynamic locomotion," in Proceedings of the 2015 IEEE International Conference on Information and Automation, ICIA 2015 - In conjunction with 2015 IEEE International Conference on Automation and Logistics, pp. 38-43, August 2015.

[12] D. Lakatos, A. Albu-Schäffer, C. Rode, and F. Loeffl, "Dynamic bipedal walking by controlling only the equilibrium of intrinsic elasticities," in Proceedings of the 16th IEEE-RAS International 
Conference on Humanoid Robots, Humanoids 2016, vol. 2016, pp. 1282-1289, IEEE, November 2016.

[13] M. A. Sharbafi, C. Rode, S. Kurowski et al., "A new biarticular actuator design facilitates control of leg function in BioBiped3," Bioinspiration \& Biomimetics, vol. 11, no. 4, Article ID 046003, 2016.

[14] N. Ryuma, N. Satoshi, and K. Yasuo, "Athlete Robot with Applied Human Muscle Activation Patterns for Bipedal Running," in Proceeding of International Conference on Humanoid Robots (Humanoids), IEEE, 2010.

[15] N. Ryuma, N. Satoshi, and K. Yasuo, "Biomechanical Approach to Open-Loop Bipedal Running with a Musculoskeletal Athlete Robot," Journal of Advanced Robotics, vol. 26, no. 3-4, pp. 445452, 2012.

[16] G. Garofalo, C. Ott, and A. Albu-Schäffer, "Walking control of fully actuated robots based on the bipedal SLIP model," in Proceedings of International Conference on Robotics and Automation (ICRA), vol. 2012, pp. 1456-1463, IEEE.

[17] Y. Liu, P. M. Wensing, D. E. Orin, and Y. F. Zheng, "Dynamic walking in a humanoid robot based on a 3D Actuated DualSLIP model," in Proceedings of the 2015 IEEE International Conference on Robotics and Automation, ICRA 2015, pp. 5710-5717, May 2015.

[18] M. Shabazi, R. Babuska, and G. A. D. Lopes, "Unified Modeling and Control of Walking and Running on Inverted Pendulum," IEEE Transactions of Robotics, vol. 32, no. 5, pp. 1178-1195, 2016.

[19] A. Kulkarni and A. Kumar, "Adaptive hierarchical control for a class of MIMO uncertain underactuated systems," in Proceedings of the 5th IEEE International Conference on Computational Intelligence and Computing Research, IEEE ICCIC 2014, pp. 305308, December 2014.

[20] D. Qian, J. Yi, and D. Zhao, "Hierarchical sliding mode control for a class of simo under-actuated systems," Control and Cybernetics, vol. 37, no. 1, pp. 159-175, 2008.

[21] D. Qian and J. Yi, Book of Hierarchical Sliding Mode Control for Under-actuated Cranes: Design, Analysis and Simulation, Springer, 2015.

[22] F. Baklouti, S. Aloui, and A. Chaari, "Adaptive Fuzzy Sliding Mode Tracking Control of Uncertain Underactuated Nonlinear Systems: A Comparative Study," Journal of Control Science and Engineering, vol. 2016, Article ID 9283103, 12 pages, 2016.

[23] K. Rsetam, Z. Cao, and Z. Man, "Hierarchical sliding mode control applied to a single-link flexible joint robot manipulator," in Proceedings of the 2016 International Conference on Advanced Mechatronic Systems, ICAMechS 2016, pp. 476-481, December 2016.

[24] A. Castigliano and E. S. Andrews, Elastic Stresses in Structures, Cambridge University Press, Cambridge, UK, 2014.

[25] T. Zielinska, "Gait Rhythm Generators of a Two-Legged Walking Machine," in Proceedings of the ROMANSY 11, Series of International Centre for Mechanical Sciences, vol. 318, pp. 165171, Springer, 1997.

[26] G. Endo, J. Morimoto, J. Nakanishi, and G. Cheng, "An empirical exploration of a neural oscillator for biped locomotion control," in Proceedings of the 2004 IEEE International Conference on Robotics and Automation, pp. 3036-3042, IEEE, May 2004.

[27] D. Hein, M. Hild, and R. Berger, "Evolution of Biped Walking Using Neural Oscillators and Physical Simulation," in Part of the Lecture Notes in Computer Science book series (LNCS), vol. 5001, pp. 433-440, Springer, 2007. 


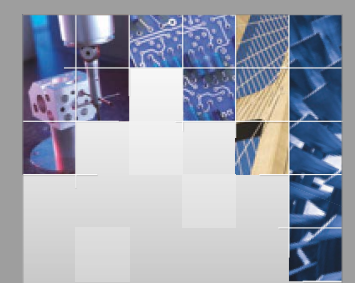

\section{Enfincering}
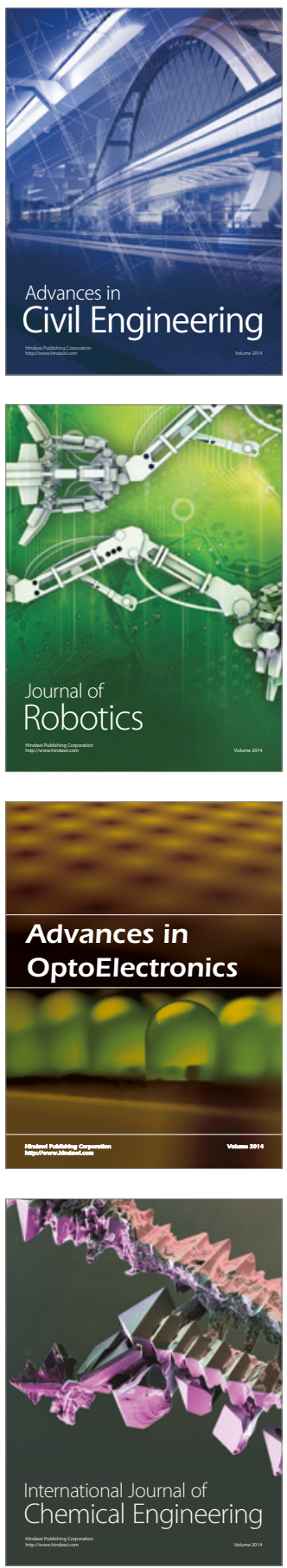

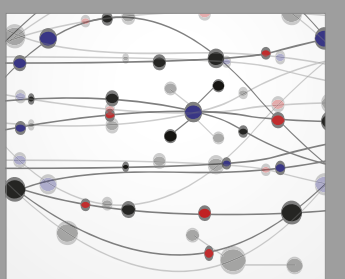

The Scientific World Journal

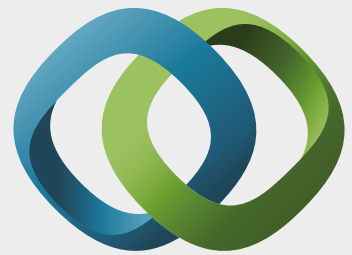

\section{Hindawi}

Submit your manuscripts at

https://www.hindawi.com
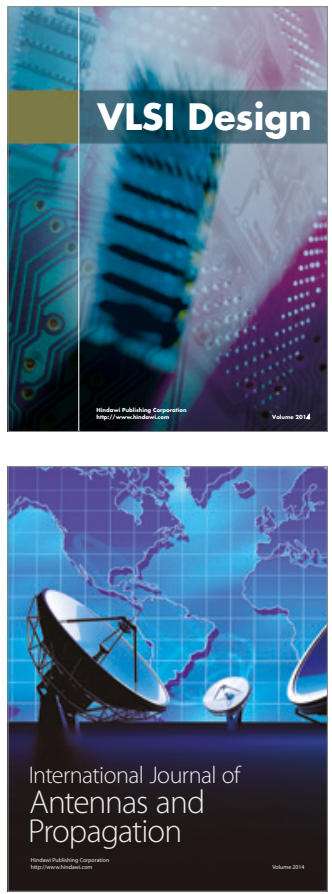

\section{Rotating}

Machinery
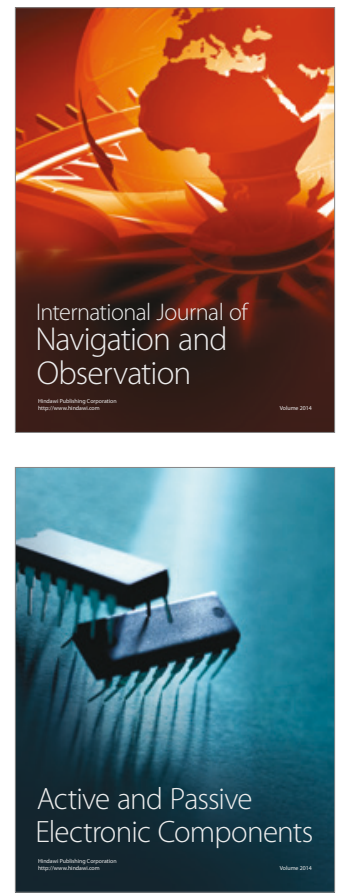
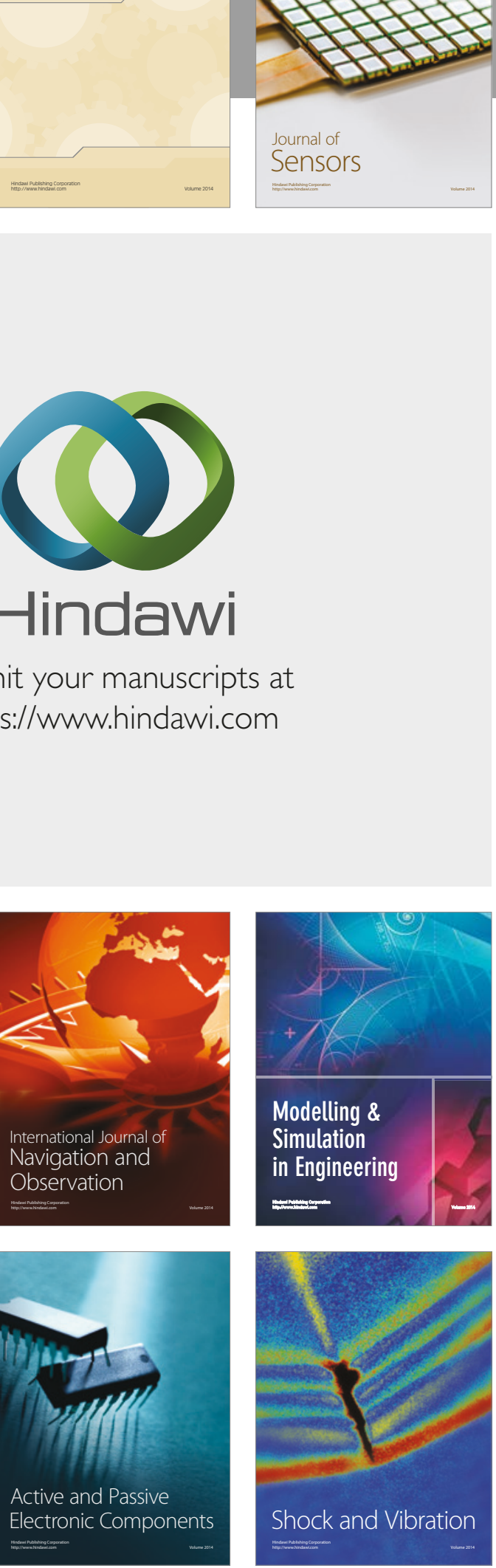
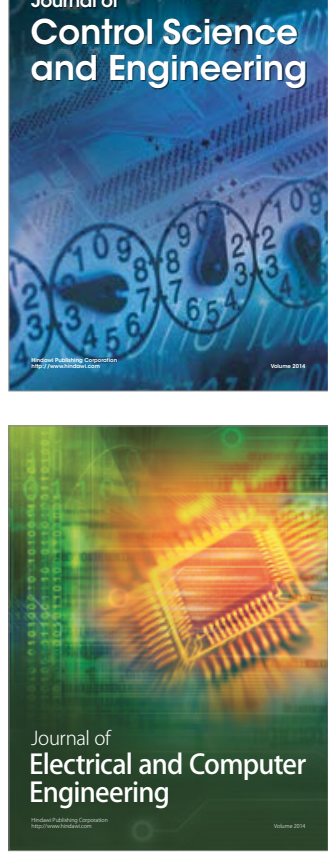

Distributed

Journal of

Control Science

and Engineering
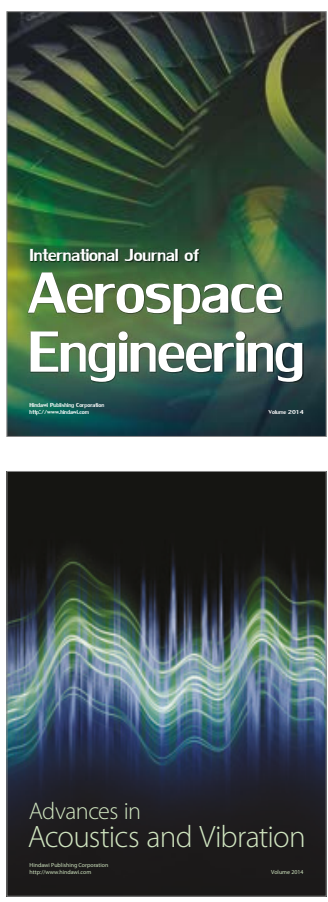

Sensor Networks 